\title{
A Cost-Effective Mobility Modelling in Nested Network Mobility ${ }^{\star}$
}

\author{
Hye-Young Kim \\ Department of Computer Science and Engineering, \\ Wright State University, Ohio, U.S.A. \\ hyeyoung.kim@wright.edu
}

\begin{abstract}
A mobile network is an entire network, moving as a unit, which dynamically change its point of attachment to the Internet and its reach-ability in the topology. Network Mobility is thus the opportunity to realize the Ubiquitous Internet, i.e. permanent access anywhere at anytime, in fixed locations and while on the move, provided that any available access network can be accommodated. In this paper, we propose a cost-effective mobility modelling in nested Network Mobility which is based on binding update multi-cast by various mobility patterns of mobile nodes in nested Network Mobility. This proposed scheme is combined Hierarchical Mobile IPv6 with Network Mobility because a mobile networks and mobile nodes move in tandem and create a hierarchy in the wireless network to management of micro-mobility and seamless handoff. These issues are focuses on the area of mobility modelling in nested Network Mobility. We address the key function for our proposed scheme and simulate the usefulness of our proposed method using mathematically analyze. We then testify that the proposal has best performance compared with Network Mobility protocol.
\end{abstract}

\section{Introduction}

In wireless mobile network, the cost of binding update messages are more challenging than in wired networks due the scarcity of bandwidth in wireless links. In our opinion, a scalable QoS protocol in Network Mobility should provide hierarchical and prefix scope binding update schemes that avoid excessive signaling for wireless network, and also it is suitable for macro mobility.

As mentioned in [1], from the routing aspect, identification between nodes is necessary for routing inside the network. A network can be virtually recognized as a single node with one address such as a prefix address and some interfaces attached to it. The single node that manages mobile network's movement is called Mobile Routers (MRs). The MR is also the default gateway for the mobile network. So, NEtwork MObility (NEMO) is transparent to the mobile inside the mobile network. So it requires changes to the binding cache of Home Agent (HA).

In the above description, we find the hierarchical relationship rooted at stationary router in the NEMO, it has an aggregated hierarchy of mobile networks,

\footnotetext{
* This work was supported by the Korea Research Foundation Grant funded by the Korean Government(MOEHRD). (KRF-2005-214-D00339).
} 


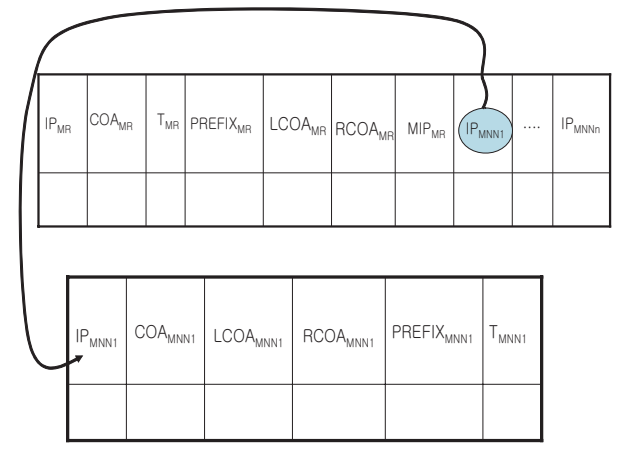

Fig. 1. The Elements in an MR

so the hierarchical mobility management is well applicable for macro mobility. Therefore, we propose a system protocol that combines Hierarchical Mobile IPv6 (HMIPv6) and NEMO which is based on prefix scope binding update protocol in nested Network Mobility.

The remainder of this paper is organized as follows. This introduction section is followed by main body of the paper, we explain a new model of integrated location management for Qos in nested Network Mobility, including a prefix scope binding update scheme and its algorithm. Section 3 presents the mathematical modelling, its simulation, and a comparison of performance between the existing NEMO and our proposed new model.

\section{Proposed System Protocol}

\subsection{Proposed System Architecture}

As motivation of our proposal described in section 1 we propose a new binding update scheme that combines HMIPV6 and NEMO for more integrated binding update. Also, Figure 1 shows the entry of the MR in this paper. $I P_{M R}$ and $I P_{M N N}$ represent MR's IP address and MNN's IP address respectively; $C O A_{M R}$ and $C O A_{M N N}$ represent MR's and MNN's COA respectively; PREFIX $X_{M R}$ and $P R E F I X_{M N N}$ represent MR's and MNN's PREFIX address respectively; $T_{M R}$ and $T_{M N N}$ represents MR's and MNN's binding lifetime respectively and $M I P_{M R}$ is a multicast address of MR. We then will explain about our proposed system structure and a scheme of combine HMIPv6 and NEMO and prefix scope bing update in this section.

Combine HMIPv6 and NEMO. Our proposed protocol combines HMIPv6 with NEMO to support multicast and locality management. As showed in the Figure 2, the MNN attaches to the MR, and the MR attaches to the MAP which is newly introduced in HMIPv6. The entire mobile network becomes a local MAP domain. The MAP advertises an address on its sub-net, $R C O A_{M R}$ 's prefix, in an RA which is a local MAP domain. The MR adds an address in its sub-net 
to its RA as optional information by using the MAP option field. All MRs and MNNs in the mobile network bind a Regional Care-of-Address (RCOA) based on mobile network prefix of MAP and bind on-Link Care-of Address (LCOA) based on the mobile network prefix of its MR 22. The MNN creates its COA based on the RA, which gives the location of the MNN in the mobile network. The MNN also creates its own $C O A_{M N N}$ with a prefix equal to PREFIX $X_{M N N}$.

When an MR/MNN moves to another mobile network, the MR/MNN receives RA containing a new MAP option, it must have a new RCOA and a new LCOA. Then, the MR must update the binding with new MAP, HA, and CNs. But if the MR/MNN doesn't change its point of attachment and receives a new MAP option, it is not necessary to update binding a new LCOA and RCOA. So, it permits a reduction in the amount of BUs message sent to CNs and HA. However, the important point is in the HMIPv6, packets must be tunnelled from the MAP to the MR and MNN. If the MAP domain is a very large-scale public network, this inefficient routing mechanism causes QoS deterioration. So, scalability becomes a more important point for NEMO.

Binding Update Multicast. In this paper, we propose a scheme based on multicast routing protocol for delivering $\mathrm{BU}$ to each $\mathrm{CNs}$ 3. In the nested NEMO, the MR which is boarder of the nest NEMO sends periodic BU containing a binding between its prefix, PREFIX $X_{M R}$, and its COA, COA $A_{M R}$, to the multicast address through its MAP. The MR uses its local MAP as an anchor point for multicast communication. All multicast traffic is directed through the MAP using RCOA as the multicast source address. The traffic forwarding between an $\mathrm{MNN} / \mathrm{MR}$ and its MAP is done using a bi-directional tunnel 4 .

If an MNN/MR moves within its MAP domain, it only registered its new LCOA using prefix scope binding update with the MAP and its parent MRs as described in section 2.1] It doesn't need to send BU message to it's HA and CNs. Therefor, it doesn't affect multicast routing trees. The movement of MNN/MR within a MAP domain remains transparent to multicast routing.

When moving into a new MAP domain, an MNN or MR with alone or nested MNN/MRs will be eager to sustain multicast connectivity via its previously established MAP. Therefore, it will learn of a new MAP through router advertisements with MAP option messages, and will perform a reactive handover. The $\mathrm{MNN} / \mathrm{MR}$ sends a BU message with its new LCOA to the previous MAP in addition to regular HMIPv6 procedures. Then, the previous MAP redirects multicast packets to new LCOA of the MNN/MR. The MNN/MR immediately should join the multicast group through the new MAP using IPv6 multicast mechanisms. And then, the multicast group traffic arrives the MNN/MR should send a $\mathrm{BU}$ message with zero lifetime to its previous MAP to eliminate its binding cache entry and end packet forwading. As we described in above, in a foreign MAP domain an MNN/MR initiates communication based on multicast by sending packets through its MAP using RCOA as its source address. MR/MNN sends the prefix scope $\mathrm{BU}$ message to a multicast group to which CNs would have subscribed through it's MAP. At the same time, it alleviates the need for the $\mathrm{MR}$ to know the list of CNs since the source of a multicast datagram does not 


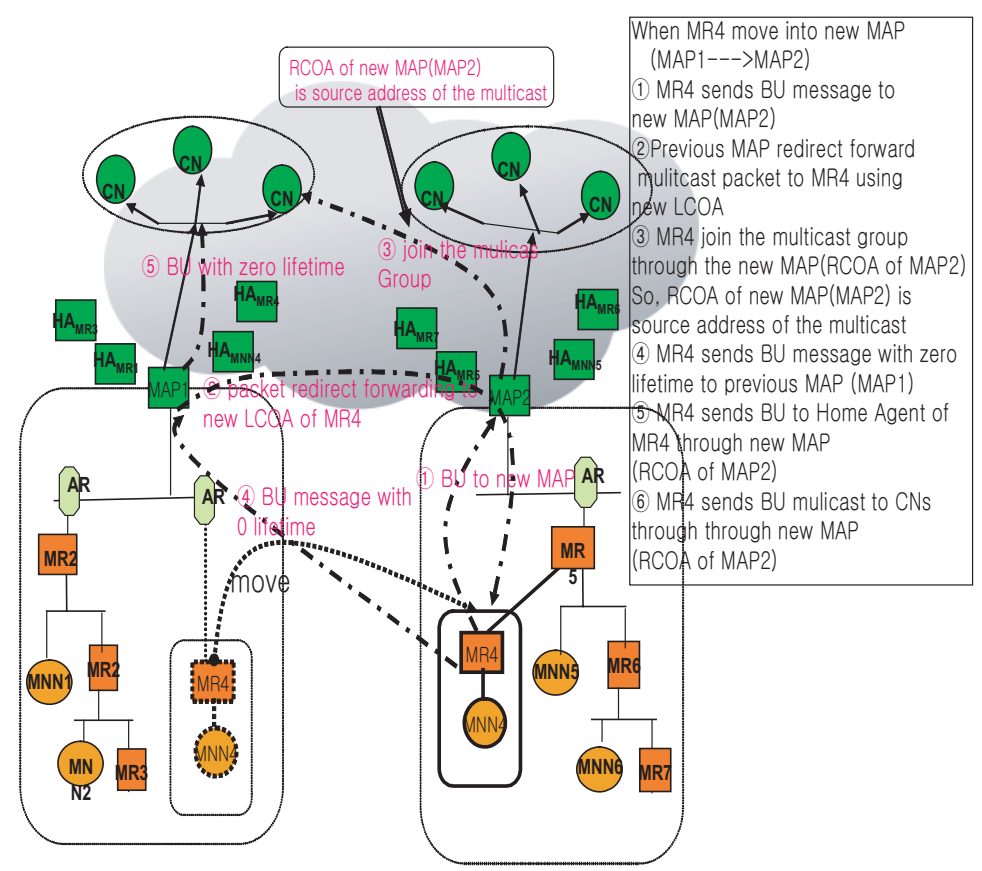

Fig. 2. Proposed Binding Update Multicast

need to know who the particular group members are. CNs join the multicast group using IPv6 multicast mechanisms. As receivers, CNs are aware of source addresses and as the RCOA may change, a HOME ADDRESS DESTINATION option must be included. By transmitting multicast packets along this path, a routing tree originating at the MAP will be constructed. The binding update instructs CNs to add an entry in their binding cache. Before sending a datagram, the $\mathrm{CN}$ checks if the prefix of the destination address matches the PREFIX $X_{M R}$ recorded in the binding cache. If so, datagrams are sent via the $C O A_{M R}$ using a Routing Extension Header. At last, authentication is made easier since BU containing the COA are always sent by the sole MR through it's MAP to the same multicast address. It might also reduce signaling and memory requirement when CNs have several correspondents in the same mobile network.

Figure 2 is a simple pictorial representation of above explanation.

\subsection{Proposed Procedures of Binding Update}

In this section, we will explain the example MR's movement and detailed procedure of prefix scope binding update multicast. In our proposed scheme, we consider a mobility of MNN and MR that is classified with Figure 3 . 


\begin{tabular}{|c|l|l|}
\hline Who where & Within MAP & Out of MAP \\
\hline $\begin{array}{l}M \\
R\end{array}$ & $\begin{array}{l}\text { MR alone } \\
\text { move }\end{array}$ & Case I-1 \\
$\begin{array}{l}\text { With MNN and } \\
\text { nested MR }\end{array}$ & Case I-2 & Case II-1 \\
\hline MNN & Case III & Case II-2 \\
\hline
\end{tabular}

Fig. 3. Classify of the MNN/MR's Mobility Pattern

\section{Performance Analysis}

In this section, we make a performance analysis of the proposed scheme in section 2 .

\subsection{An Analytic Mobility Modelling of Binding Update Cost in the Proposed Scheme}

This section gives the performance analysis, in which we compute the average binding update cost per unit time for a single MNN/MR and nested MRs to examine the effects of the proposed in Section 2. Specially we computed the reduction in the mean binding update cost and amount of traffic through the MNNs and MRs in the whole network of the nested Network Mobility.

The binding update cost in nested NEMO effect due to the proposed method was evaluated in terms of both the hierarchical number of the nested mobile network and network scale. We describe a two-dimensional random walk model for mesh planes in order to compute the MAP residence probability of an MR and MNN. We assume that an MR resides in a MAP domain for a period and moves to one of its four neighbors with equal probability. We assume the service domain of a MAP is sub-netted as shown in Figure 4. These sub-nets form many rings, each of which consists of a circle of rectangles. We label the outermost ring in the area with " 1 ", which is denoted by $b_{1}$. Similarly, we label the next inner ring with "2", which is denoted $b_{2}$ and so forth. We assume that there are $n$ rings in a MAP domain. The label number represents the distance from a sub-net to the border of the MAP. For example, ring $b_{3}$ consists of all the sub-nets with a distance of 3 to the border. To derive the probability of an $\mathrm{MR}$ residing at a ring, first we calculate the number of sub-nets in a ring. We suppose that the length and width of a MAP are $L$ and $W$ grid, respectively. $N\left(b_{i}\right)$ denotes the number of grids in the $i^{\text {th }}$ ring. Thus, $N\left(b_{i}\right)$ can be computed as $N\left(b_{i}\right)=2 L+2 W-8_{i}+4(i \geq 1)$. Therefore, the number of $n^{\text {th }}$ rings can be obtained from the given length and width as

$$
n=\min \left\{\left\lceil\frac{L}{2}\right\rceil,\left\lceil\frac{W}{2}\right\rceil\right\}
$$

In Figure 4, for example $L=7$ and $W=6$, we get $n=3, N\left(b_{1}\right)=22, N\left(b_{2}\right)=14$, and $N\left(b_{3}\right)=6$. Therefore $S$ denotes the number of grids in the MAP as follows:

$$
S=\sum_{i=1}^{n} N\left(b_{i}\right)
$$




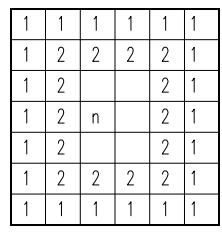

Fig. 4. The Grid Structure of an MAP

Hence, the case where an MR escapes from the MAP is a located boundary, which we denote by $\mathrm{X}$ and has the following rate:

$$
\left.X=\left[\frac{1}{4}\left(N\left(b_{1}\right)-4\right)+4 \cdot \frac{1}{2}\right]=\left[\frac{1}{4} N\left(b_{1}\right)+1\right)\right]
$$

Let $P(\alpha)$ and $P(\beta)$ be the probability for an MR and MNN moving to a neighbor RA during unit time, respectively. we denote $\pi(r)$ as the probability that an MR will leave the MAP domain alone or with nested MNN and MRs as follows:

$$
\pi(r)=\frac{X}{S} \cdot P(\alpha)
$$

Similarly, we denote $\widehat{\pi(r)}$ as the probability that an MR will stay within the MAP domain as follows:

$$
\widehat{\pi(r)}=1-\pi(r)
$$

In NEMO protocol, the mobility of an MNN is influenced by the mobility of an MR. Therefore, the movement of an MNN can happen when an MR does not move. As explained in section 3, since MR movements accompany MNN movements, the probability of MR movement is equal to that of MNN movements. Here an MNN moves within or out of the MAP. We denote $\pi(n)$ as the probability that an MNN will leave the MAP domain as follows:

$$
\pi(n)=\left(\frac{X}{S} \cdot X\right) \cdot(1-P(\alpha)) \cdot P(\beta)
$$

Therefore, probability $\widehat{\pi(n)}$ whereby an MNN stays in MAP is denoted as follows:

$$
\widehat{\pi(n)}=(1-P(\alpha)) \cdot P(\beta) \cdot(1-\pi(n))
$$

Here, we use a parameters such as Table 1 for calculate of a binding update cost of the MR and MNN. If the MNN moves within the MAP, it is referred to as $C_{M N N_{-} \text {within_MAP }}$; if the MNN moves out of the current the MAP, it is referred to as $C_{M N N_{-} o u t} M A P$; if the MR moves within the same MAP or expires just before lifetime of MR, it is referred to as $C_{M R_{-} \text {within_MAP }}$; and if the MR moves into another MAP, it is referred to $C_{M R_{-} o u t_{-} M A P}$. The following computations can apply to each case for a one-time binding update cost. Since usually the costs 
Table 1. The Parameters for Binding Update Cost

\begin{tabular}{|l|l|}
\hline Parameter & Description \\
\hline \hline$C_{m r}$ & The transmission cost of location between the MNN and the MR. \\
\hline$C_{r p}$ & The transmission cost of location between the MR and the MAP. \\
\hline$C_{r h r}$ & The transmission cost of location between the MR and the MR's HA. \\
\hline$C_{r h n}$ & The transmission cost of location between the MR and the MNN's HA. \\
\hline$C_{p h r}$ & The transmission cost of location between the MAP and the MR's HA \\
\hline$C_{p c}$ & The transmission cost of location between the MAP and the CNs. \\
\hline$C_{p h n}$ & The transmission cost of location between the MAP and the MNN's HA. \\
\hline$C_{r c}$ & The transmission cost of location between the MR and the CNs. \\
\hline$C_{h n}$ & The transmission cost of location between the MR's HA and the MNN's HA. \\
\hline$C_{r m}$ & The transmission cost of location between the MR and the MNNs. \\
\hline$C_{h c}$ & The transmission cost of location between the MR's HA and the CNs. \\
\hline$C_{h m}$ & The transmission cost of location between the MR'HA and the MAP. \\
\hline$U_{m r}$ & The processing cost of location update at the MR. \\
\hline$U_{m a p}$ & The processing cost of location update at the MAP. \\
\hline$U_{h m r}$ & The processing cost of location update at the MR's HA. \\
\hline$U_{h m n}$ & The processing cost of location update at the MNN's HA. \\
\hline$U_{c n}$ & The processing cost of location update at the CNs. \\
\hline
\end{tabular}

for a wireless communication network are higher than for a wire communication network, the ratio is considered by using $\rho$ to distinguish the difference between wire and wireless communication networks ( $\rho$ is dependent on the network, which is $\rho \geq 1)$. The communication cost for a wire communication network is referred to as $\delta$, and the transmission cost for a wireless communication is referred to as $\delta \rho$. The multicast Listener Discovery (MLD) protocol is used by IPv6 multicast routers to learn about the existence of multicast group members on their connected links. We applied multicasting to binding updates that send to CNs in this paper. Therefore, we consider using $\gamma$ as the cost of multicast in our proposed scheme. $c$ and $m$ represent the number of the MNN's $\mathrm{CN}$ and the number of MNN within the MR, respectively. $C_{m r}, C_{r p}, C_{r h r}, C_{p c}, C_{r m}, C_{r h n}$, and $C_{r c}$ are the wireless communication networks that incur most expensive communication costs. Because the number of hops or the distance between routers does not have much influence on the number of BU messages or on costs, they are not considered in this paper. The following computations can be applied to each case for the one-time binding update cost.

$$
\begin{aligned}
& C_{M N N_{\text {_out_MAP }}}=(6+\gamma) \delta \rho+U_{m r}+U_{m a p}+U_{h m r}+\gamma \rho+c \cdot U_{c n} \\
& C_{M R_{-} o u t_{-} M A P}=(4+2 \gamma) \delta \rho+U_{m a p}+U_{h m r}+c \cdot U_{c n}+m \cdot U_{h m n} \\
& C_{M N N \_w i t h i n \_M A P}=4 \delta \rho+U_{m r}+U_{\text {map }} \\
& C_{M R_{-} \text {within_MAP }}=3 \delta \rho+U_{\text {map }}
\end{aligned}
$$

Thus, we can get the total binding update cost, $C_{B U}$ in the proposed scheme from formulae (5) and (11) - (4) as follows:

$$
\begin{aligned}
C_{B U} & =\pi(r) \cdot C_{M R_{-} o u t \_M A P}+\widehat{\pi(r)} \cdot C_{M R_{-} i n_{-} M A P}+\pi(n) \cdot C_{M N N_{-} o u t_{-} M A P} \\
& +\widehat{\pi(n)} \cdot C_{M N N_{-} o u t_{-} M A P .}
\end{aligned}
$$




\subsection{Comparison and Analysis Between Proposed Scheme and NEMO}

We have analyzed the binding update costs of MR and MNN depending on the number of CNs, the number of MNNs within an MR, and the multicast cost ratio compared them with NEMO. The results were computed by MATLAB, and Table 2 lists some of the parameters used in our performance analysis.

Table 2. Performance Analysis Parameter

\begin{tabular}{|l|l|l|l|l|l|l|l|l|l|l|l|l|l|}
\hline$U_{m r}$ & $U_{\text {map }}$ & $U_{h m r}$ & $U_{h m n}$ & $U_{c n}$ & $\delta$ & $\rho$ & $\gamma$ & $P(\alpha)$ & $P(\beta)$ & $\mathrm{M}$ & $\mathrm{C}$ & $\mathrm{W}$ & $\mathrm{L}$ \\
\hline 10 & 15 & 25 & 25 & 25 & 1 & 10 & $0.1-1$ & $0.3-0.8$ & $0.1-0.6$ & 20 & 20 & 6 & 7 \\
\hline
\end{tabular}

In Table 2, $m$ represents the number of MNNs within an MR, and $c$ represents the maximum number of $\mathrm{CNs} ; \rho$ is a variable that distinguishes the difference in communication costs between wire and wireless communication networks. The length and width of a MAP are $L$ and $W$ grid, and they are 7 and 6 , respectively.

$\gamma$ is a variable for multicast costs in network. We propose a scheme based on multicast routing protocols for delivering binding update to each $\mathrm{CN}$. There are many multicast routung protocols such as DVMRP, PIM-DM, and CBT etc. Our propose scheme is influenced to multicast routing protocol and membership mechanism such as joining, leaving, and sending data to a multicat group. Also, to analyze the location management costs according to multicast ratio, $\gamma$ is changed for the binding update cost in the case of 1, 0.5, 0.2. 0.1. In Figure 5, the smaller multicost ratio ( NEMO vs the Proposed Scheme ) is, the greater the differences between NEMO and our method is. $U_{m r}, U_{m a p}, U_{h m r}, U_{h m n}$ and $U_{c n}$ mean costs for location update performance from MR, MAP, MR's HA, MNN's HA and the CNs, respectively. $P(\alpha)$ and $P(\beta)$ represent the probability of probability for $\mathrm{MR}$ and MNN move by neighbor RA during unit time, respectively. Here, we assume $P(\alpha) \geq P(\beta)$. To analyze the binding update costs according to mobility of MR

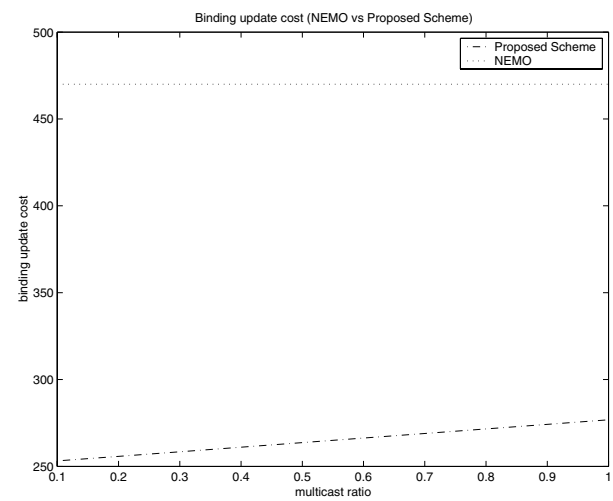

Fig. 5. According to Multicast Ratio 


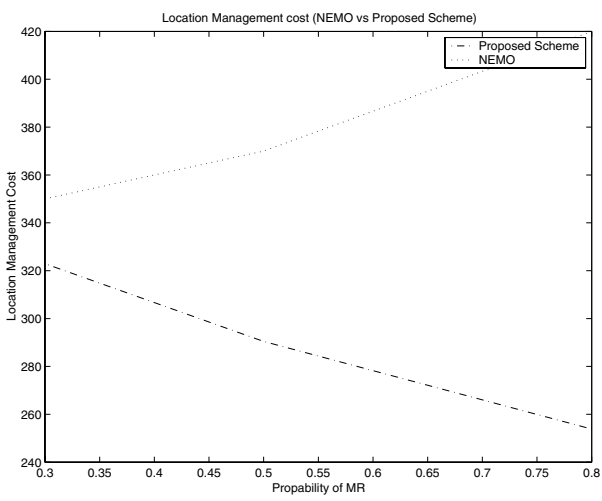

Fig. 6. According to $P(\alpha)$

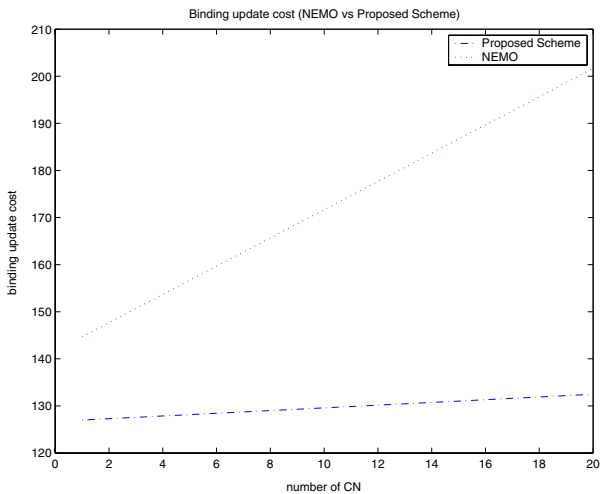

Fig. 7. According to Number of CNs

and MNN, $P(\alpha)$ and $P(\beta)$ are assigned to $0.8,0.5$.and 0.3 and $0.1,0.4,0.6$, respectively. As in Figure 6, our technique is more efficient when the mobility of MR becomes greater. The binding update costs of our scheme have been computed by increasing the number of CNs from 1 to 20.

\section{Conclusion}

One of the most important and challenge problems in mobile communication is mobility management. To support comprehensive and stable system performance and quality with seamlessly integrated and converged with Mobile IP-based networks, we propose a scheme which is an integrated binding update method for each case where binding updates of MNNs and MRs happen, and we calculate the location management costs required in each case. Further presented in this paper is a technique that can minimize binding update costs for MNN and MR by applying the binding update multicasting for CNs. Finally, we calculate binding 
update costs for each technique of NEMO protocol and our proposed method and perform a comparison analysis. Our proposed method is remains almost constantly without being influence by the increased number of MNNs and CNs. As shown in section 3, our technique is more efficient when the mobility of MR becomes greater.

Consequently, when compared with NEMO protocol, our proposed scheme is useful, especially when the number of MNNs or CNs increases and MR moves frequently.

\section{References}

1. Vijay Devarapalli, Ryuii Wakikawa, Alexandru Petrescu, Pascal Thubert, "Network Mobility(NEMO) Basic Support Procol", Requesut For Commnets 3963, Internet Engineering Task Force(IETF), January 2005.

2. H.Soliman and C.Castelluccia and K. El-malki, and L. Beller, "Hierarchical MIPv6 mobility management (HMIPv6)", dfraft-ietf-mipshop-hmipv6-04.txt, December 2004.

3. H-Y. Kim, "An Integrated Location Management Scheme in the Network Mobility", Ph.D thesis, Department of Computer Science and Engineering, Korea University, Dec. 2004.

4. Conta, A., Deering, S., "Generic Packet Tunneling in IPv6 Specification", IETF Request For Commnents 2473, December 1998. 